The Astrophysical Journal, 550:547-553, 2001 April 1

(C) 2001. The American Astronomical Society. All rights reserved. Printed in U.S.A.

\title{
DETERMINING THE GEOMETRY AND THE COSMOLOGICAL PARAMETERS OF THE UNIVERSE THROUGH SUNYAEV-ZELDOVICH EFFECT CLUSTER COUNTS
}

\author{
Zuhui FAN ${ }^{1,2,3}$ AND TzIHONG CHIUEH ${ }^{2,3}$ \\ Received 2000 May 25 ; accepted 2000 November 15
}

\begin{abstract}
We study Sunyaev-Zeldovich effect (SZE) cluster counts in different cosmologies. We find that even without full knowledge of the redshift distribution of SZE clusters, one can still readily distinguish a flat universe with a cosmological constant from an open universe. We divide clusters into a low-redshift group (with redshift $z \leq 0.5$ ) and a high-redshift group (with $z \geq 1$ ), and compute the ratio of $r=N(z \leq 0.5) / N(z \geq 1)$, where $N(z \leq 0.5)$ is the number of flux-limited $\left(S_{v}^{\lim }\right)$ SZE clusters with $z \leq 0.5$, and $N(z \geq 1)$ is the number of flux-limited SZE clusters with $z \geq 1$. With about the same total number of SZE clusters $N(z \geq 0)$, the $r$ values for a flat universe with a nonzero cosmological constant and for an open universe occupy different regions in the $S_{v}^{\text {lim }}-r$ plot for the most likely cosmological parameters $0.25 \leq \Omega_{0} \leq 0.35$ and $0.2 \leq \Gamma \leq 0.3$, where $\Omega_{0}$ is the matter density parameter of the universe and $\Gamma$ is the shape parameter of the power spectrum of linear density fluctuations. Thus, with a deep SZE cluster survey, the ratio $r$ can reveal independently of the normalization of the power spectrum whether we are living in a low-density flat universe or in an open universe. Within the flat universe scenario, the SZE cluster-normalized $\sigma_{8}$ is studied, where $\sigma_{8}$ is the rms density fluctuation within the top-hat scale $8 \mathrm{Mpc}$ $h^{-1}$, where $h$ is the Hubble constant in units of $100 \mathrm{~km} \mathrm{~s}^{-1} \mathrm{Mpc}^{-1}$. A functional relation of $\sigma_{8} \propto \Omega_{0}^{-0.13}$ is found. Combined with the X-ray cluster-normalized $\sigma_{8} \propto \Omega_{0}^{-0.52+0.13 \Omega_{0}}$, one can put constraints on both $\Omega_{0}$ and $\sigma_{8}$ simultaneously.

Subject headings: cosmology: theory — galaxies: clusters: general — large-scale structure of universe
\end{abstract}

\section{INTRODUCTION}

Clusters of galaxies are the largest virialized objects in the universe, and contain valuable information about the universe and its large-scale structure. There have been intensive studies of clusters using different approaches, such as strong and weak gravitational lensing, and X-ray and optical observations. With the technical advent of interferometers, the cluster's Sunyaev-Zeldovich effect (SZE; Sunyaev \& Zeldovich 1970, 1980; Birkinshaw 1999; Carlstrom et al. 1999), a spectral distortion of cosmic microwave background radiation $(\mathrm{CMB})$ due to scattering of $\mathrm{CMB}$ photons by hot electrons within clusters, has been becoming a new probe for cluster study. As CMB photons pass through intracluster hot electrons, on average they gain energies through inverse Compton scattering, and as a result of this, the number of low-energy photons decreases while the number of high-energy ones increases. Thus, for observations with the frequencies $v>219$ or $v<219 \mathrm{GHz}$, hot clusters behave like emitting sources or absorbers, respectively, of photons. The equivalent temperature increment (or decrement) $\Delta T$ of CMB photons toward clusters is proportional to $\int n_{e} T_{\mathrm{gas}} d l$, where $n_{e}$ is the number density of electrons, $T_{\text {gas }}$ is the hot gas temperature, and $d l$ is the line element along the line of sight. The integrated SZ effect of a cluster is then directly proportional to the cluster's gas mass if the gas is close to isothermal. Therefore, the integrated SZ effect is not sensitive to the lumpy structures of the gas, and the gas fraction can be estimated relatively cleanly from the SZ effect in conjunction with lensing observations (e.g.,

\footnotetext{
${ }^{1}$ Department of Astronomy and Astrophysics, University of Chicago, 5640 South Ellis Avenue, Chicago, IL 60637.

2 Department of Physics, National Taiwan University, 1 Roosevelt Road, Section 4, Taipei, Taiwan, Republic of China.

${ }^{3}$ Institute of Astronomy and Astrophysics, Academia Sinica, P.O. Box 1-87, Nankang, Taipei 115, Taiwan, Republic of China.
}

Grego et al. 2000). On the other hand, because of the different dependence on $n_{e}$ of the cluster's X-ray surface brightness $\left(S_{\mathrm{X}} \propto \int n_{e}^{2} \Lambda_{e H} d l\right.$, where $\Lambda_{e H}$ is the X-ray cooling function) and of the SZ effect, the angular diameter distance to a cluster can be derived directly from a joint analysis of the X-ray emission and the SZ effect by modeling the gas density profile properly (e.g., Reese et al. 2000).

Apart from the SZE studies for individual clusters, statistical investigations of a large number of SZE clusters can also yield very promising results. In fact, several interferometric arrays have been proposed for surveying SZE clusters, including the Array for Microwave Background (AMIBA) project, which has been established in Taiwan. Because of the frequency (energy) redshift dependence of the $\mathrm{CMB}$ photons, the SZ effect is independent of the redshift, which permits a SZE cluster survey to detect very high redshift clusters with relative ease. Therefore, the cluster redshift evolution can be studied with high statistical significance. Another advantage of the SZE cluster over X-ray studies is that the integrated SZ effect of an individual cluster is directly proportional to the gas mass within the cluster (assuming that the gas is isothermal), which is in turn related to the total mass of the cluster, and the number of SZE clusters can be predicted analytically from the PressSchechter formula (or other similar models) in a straightforward manner with certain qualifications. By contrast, because of the $n_{e}^{2}$ dependence of the X-ray surface brightness, the gas density profile must be modeled in the X-ray studies to estimate flux-limited X-ray cluster number counts analytically, a procedure that can introduce large uncertainties (the prediction of the number of temperaturelimited X-ray clusters suffers fewer problems). One can investigate both aspects of clusters with numerical simulations, but finite numerical resolutions and box sizes ultimately limit their application, and the analytical analysis can be complementary. 
Among other promising applications, the redshift distribution of SZE clusters can be used to constrain cosmological parameters. The redshift distribution of SZE clusters with $\Omega_{0}=1$ is distinctly different from those with $\Omega_{0} \sim 0.3$, and the existence of several SZE clusters at redshift $z \gtrsim 1$ would strongly exclude the $\Omega_{0}=1$ model. The difference between the redshift distribution of a low-density flat universe model and that of a low-density open universe model is less dramatic, and one needs a relatively large number of clusters at high redshift to falsify them. The SZE cluster surveys are suitable for this purpose. By fully using the redshift distribution and the total number of SZE clusters, Haiman, Mohr, \& Holder (2000) studied constraints on the quintessence theory from future SZE (and X-ray) cluster surveys. The redshift of a cluster with $z \leq 1$ can be obtained by using the photometric method around a characteristic spectral break from full spectral analyses or if its full spectrum is hard to get. For clusters with $z>1$, however, it appears difficult to measure the redshifts precisely except for very large clusters. In this paper, we propose a method that can distinguish, within the parameter regime $0.25 \leq$ $\Omega_{0} \leq 0.35$ and $0.2 \leq \Gamma \leq 0.3$, a flat universe with a nonzero cosmological constant from an open universe, even if the full redshift distribution of SZE clusters is not known. We divide clusters into two groups: a low-redshift one with $z \leq 0.5$, and a high-redshift one with $z \geq 1$. We study the ratio $r=N(z \leq 0.5) / N(z \geq 1)$ for different cosmologies with different parameters, where $N(z \leq 0.5)$ is the total fluxlimited number of clusters with $z \leq 0.5$, and $N(z \geq 1)$ is the total flux-limited number of clusters with $z \geq 1$. We find that $r$ can be used to disentangle a low-density flat universe from a low-density open universe. Note that to compute $r$, we only need to know the redshift range of a cluster (whether it is $z \leq 0.5$ or $z \geq 1$ ) rather than its precise redshift.

We also investigate, within the framework of the flat universes with a nonzero cosmological constant, the SZE cluster-normalized $\sigma_{8}$. The $\sigma_{8}-\Omega_{0}$ relation inferred from the SZE cluster counts is distinctly different from that inferred from the X-ray cluster counts, with the latter being steeper. This difference can be used to limit the parameter regime of $\Omega_{0}$ and $\sigma_{8}$ simultaneously.

The rest of the paper is organized as follows. Section 2 presents the formulation for the study. The results are shown in $\S 3$. Section 4 contains a summary.

\section{FORMULATION}

As CMB photons pass through a sea of hot electrons, their blackbody spectrum is distorted by inverse Compton scattering. The SZ effect can be characterized by the Compton $y$-parameter,

$$
y=\int n_{e} \sigma_{\mathrm{T}}\left(\frac{k T_{\mathrm{gas}}}{m_{e} c^{2}}\right) d l,
$$

where $n_{e}$ is the number density of hot electrons, $\sigma_{\mathrm{T}}=6.65$ $\times 10^{-25} \mathrm{~cm}^{2}$ is the Thomson cross section, $k$ is the Boltzmann's constant, $T_{\text {gas }}$ is the temperature of the hot intracluster gas, $m_{e}$ is the electron mass, and $c$ is the speed of light. The integration is along the line of sight, and the $y$-parameter is proportional to the integrated thermal pressure along the line of sight. When the electron temperature $T_{\text {gas }}$ is much higher than the temperature $T_{\mathrm{CMB}}$ of the CMB photons, the CMB flux change due to the presence of a cluster can be written as

$$
S_{v}=S_{v}^{\mathrm{CMB}} Q(x) Y,
$$

where $x=h_{\mathrm{P}} v / k T_{\mathrm{CMB}}, v$ is the frequency of the CMB photons, $h_{\mathrm{P}}$ is Planck's constant, the unperturbed CMB flux $S_{v}^{\mathrm{CMB}}=\left(2 h_{p} v^{3} / c^{2}\right) /\left(e^{x}-1\right)$,

$$
Q(x)=\frac{x e^{x}}{e^{x}-1}\left[\frac{x}{\tanh (x / 2)}-4\right]
$$

and

$$
Y=R_{d}^{-2} \int y d A,
$$

where $R_{d}$ is the angular diameter distance of the cluster, and the integration is over the projected area of the cluster. It can be seen that when $v \approx 219 \mathrm{GHz}, Q(x)=0$. At the lower and higher frequency parts, $Q(x)<0$ and $Q(x)>0$, respectively. For AMIBA, $v=90 \mathrm{GHz}, x \approx 1.58$, and $Q(x) \approx-3.185$.

We assume that the intracluster gas is isothermal and the gas mass fraction $f_{\text {ICM }}$ is a constant. Then we have (e.g., Eke, Cole, \& Frenk 1996)

$$
Y=\frac{\sigma_{\mathrm{T}}}{2 m_{e} m_{p} c^{2}} R_{d}^{-2} f_{\mathrm{ICM}}(1+X) k T_{\mathrm{gas}} M,
$$

where $m_{p}$ is the proton mass, $X$ is the hydrogen mass fraction, and $M$ is the total mass (including the dark matter) of the cluster. Here we have assumed that the intracluster gas mass is dominated by hydrogen and helium. Furthermore, the gas is assumed to be in hydrostatic equilibrium with the gravitational potential of the total mass of the cluster; then

$$
k T_{\mathrm{gas}}=-\frac{1}{\left[d \ln \rho_{\mathrm{gas}}(r) / d \ln r\right]_{r_{\mathrm{vir}}}} \mu m_{p} \frac{G M}{r_{\mathrm{vir}}},
$$

where $\rho_{\text {gas }}(r)$ is the radial density profile of the gas, $r_{\mathrm{vir}}$ is the virial radius of the cluster, and $\mu=4 /(5 X+3)$ is the mean molecular weight. In equation (6) we have used the virial mass to represent the total mass of the cluster. Let $\Delta_{c}$ be the average mass density with respect to the critical density at redshift $z$ of the cluster formation; then

$$
\begin{aligned}
k T_{\text {gas }}= & -\frac{7.75}{0.5\left[d \ln \rho_{\text {gas }}(r) / d \ln r\right]_{\text {vir }}} \\
& \times\left(\frac{6.8}{5 X+3}\right)\left(\frac{M}{10^{15} h^{-1} M_{\odot}}\right)^{2 / 3} \\
& \times(1+z)\left[\frac{\Omega_{0}}{\Omega(z)}\right]^{1 / 3}\left(\frac{\Delta_{c}}{178}\right)^{1 / 3} \mathrm{keV},
\end{aligned}
$$

where $\Omega(z)$ is the density parameter at redshift $z$, and $h$ is the Hubble constant in units of $100 \mathrm{~km} \mathrm{~s}^{-1} \mathrm{Mpc}^{-1}$. Then

$$
\begin{aligned}
S_{v}= & 2.29 \times 10^{4} \frac{x^{3}}{e^{x}-1} Q(x) \times 1.70 \times 10^{-2} h \\
& \times\left(\frac{f_{\mathrm{ICM}}}{0.1}\right)\left(\frac{1+X}{1.76}\right)\left\{\frac{7.75}{0.5\left[d \ln \rho_{\mathrm{gas}}(r) / d \ln r\right]_{r_{\mathrm{vir}}}}\right\} \\
& \times\left(\frac{6.8}{5 X+3}\right)\left(\frac{R_{d}}{100 h^{-1} \mathrm{Mpc}}\right)^{-2}(1+z)\left[\frac{\Omega_{0}}{\Omega(z)}\right]^{1 / 3} \\
& \times\left(\frac{\Delta_{c}}{178}\right)^{1 / 3}\left(\frac{M}{10^{15} h^{-1} M_{\odot}}\right)^{5 / 3} \mathrm{mJy},
\end{aligned}
$$


where $1 \mathrm{mJy}=10^{-26} \mathrm{ergs} \mathrm{cm}^{-2} \mathrm{~s}^{-1} \mathrm{~Hz}^{-1}$. In the following discussion we use the value $\left[d \ln \rho_{\mathrm{gas}}(r) / d \ln r\right]_{r_{\mathrm{vir}}}=2$, which is consistent with both the observational and the numerical simulation results. In fact, the results for different values of $\left[d \ln \rho_{\text {gas }}(r) / d \ln r\right]_{r_{\text {vir }}}$ can be obtained from our analyses by rescaling the overall flux $S_{v}$ up or down, as can be seen from equation (8). The cosmology enters equation (8) between $S_{v}$ and $M$ through the angular diameter distance $R_{d}$, the density parameters $\Omega_{0}$ and $\Omega(z)$, and the overdensity parameter $\Delta_{c}$. We calculate the flux-limited SZE cluster counts. Note that by using equation (8) to calculate $M_{\text {lim }}$ from a given flux limit $S_{v}^{\text {lim }}$, we have implicitly assumed that the counts are for unresolved clusters. An array of interferometers must, however, have a minimum baseline that is essentially limited by the dish diameter $D$. Signals from angular scales larger than about $\lambda /(2 D)$ are lost where $\lambda$ is the observing wavelength. For AMIBA, there are two sets of dishes with $D=1.2$ and $0.3 \mathrm{~m}$, respectively. For $v=90$ $\mathrm{GHz}, \lambda \approx 0.33 \mathrm{~cm}$; and $\lambda /(2 D) \sim 4^{\prime} .7$ for $D=1.2 \mathrm{~m}$ and $18^{\prime} .9$ for $D=0.3 \mathrm{~m}$. For the smaller set of dishes, there should not be any loss of signal from clusters. We have estimated the mass limit for $D=1.2 \mathrm{~m}$ by simply cutting off any signals from $\theta \geq 4: 7$. The hydrostatic equilibrium gas density profile has been used by assuming that the underlying dark matter distribution has a universal density profile (Navarro, Frenk, \& White 1997). The mass limit estimated here is not very different from that for the unresolved clusters at $S_{v}^{\text {lim }} \sim$ $5 \mathrm{mJy}$ of the AMIBA design. Thus, our studies presented in the next section only consider the unresolved cluster counts. Holder et al. (2000) determined $M_{\text {lim }}$ by performing mock observations appropriate for a proposed interferometric array consisting of ten $2.5 \mathrm{~m}$ telescopes operating at $v=30$ $\mathrm{GHz}$ (Mohr et al. 1999) on simulated clusters; the shape of the $M_{\text {lim }}(z)$ is similar to that for unresolved clusters. In the work of Haiman et al. (2000), the mass limit for their fiducial model is from Holder et al. (2000), and $M_{\text {lim }}$ for other cosmological models is obtained by using the same scaling relation as that of equation (8). They found that the mass limit from the scaling relation agrees with the mock survey results to better than $10 \%$ for the two tested cosmological models. We use the Press-Schechter formalism (Press \& Schechter 1974) to calculate the number of SZE clusters. The comoving number density of clusters of mass $M$ with width $d M$ is

$$
n(M) d M=\left(\frac{2}{\pi}\right)^{1 / 2} \frac{\rho_{0}}{M} \frac{\delta_{c}(z)}{\sigma_{0}^{2}} \frac{d \sigma_{0}}{d M} \exp \left(-\frac{\delta_{c}^{2}(z)}{2 \sigma_{0}^{2}}\right),
$$

where $\rho_{0}$ is the present mass density of the universe, $\delta_{c}(z)$ is the linear overdensity threshold for collapse at redshift $z$, and $\sigma_{0}$ is the rms linear density fluctuation on the scale corresponding to $M$. Note that $\delta_{c}(z)$ and $\sigma_{0}$ are computed from the extrapolated-to-present linear density perturbations. Then the differential number of SZE clusters is

$$
\frac{d N}{d z d \Omega}=\frac{d V}{d z d \Omega} \int_{M_{\lim (z)}} n(M) d M,
$$

where $d \Omega$ is the solid angle element; $d V$ is the comoving volume element, which is dependent on cosmologies; and $M_{\text {lim }}(z)$ is calculated from equation (8).

\section{ANALYSES}

\subsection{Flat Models versus Open Models}

For a cold dark matter universe, the power spectrum of the linear density fluctuation field can be written as (e.g., Efstathiou, Bond, \& White 1992)

$$
P(k)=\frac{B k^{n}}{\left\{1+\left[a k+(b k)^{3 / 2}+(c k)^{2}\right]^{v}\right\}^{2 / v}},
$$

where $a=(6.4 / \Gamma) h^{-1} \mathrm{Mpc} ; b=(3.0 / \Gamma) h^{-1} \mathrm{Mpc} ; c=(1.7 /$ Г) $h^{-1} \mathrm{Mpc} ; v=1.13 ; \Gamma$ is the shape parameter of the power spectrum, which is related to the time of equal matter-radiation energy density in the universe; $h$ is the Hubble constant in units of $100 \mathrm{~km} \mathrm{~s}^{-1} \mathrm{Mpc}^{-1}$; $B$ represents the perturbation amplitude; and $n$ is the power index, which is taken to be $n=1$.

The object of this subsection is to distinguish the lowdensity flat universes from the low-density open universes. Before proceeding with this issue, let us first consider examples of the redshift distribution of SZE clusters for some popular cosmologies. In Figure 1, the SZE clusters' redshift distribution is shown for (1) $\tau$ CDM (White, Gelmini, \& Silk 1995; solid line): $\Omega_{0}=1, h=0.5, \Gamma=0.25$, and $\sigma_{8}=0.52$, where $\sigma_{8}$ is the rms density fluctuation within the top-hat scale $8 \mathrm{Mpc} h^{-1}$; (2) SCDM (dash-triple-dotted line): $\Omega_{0}=$ $1, h=0.5, \Gamma=0.5$, and $\sigma_{8}=0.52$; (3) open CDM (dashdotted line): $\Omega_{0}=0.3, \Omega_{\Lambda}=0, h=0.83, \Gamma=0.25$, and $\sigma_{8}=$ 0.87 ; and (4) $\Lambda \mathrm{CDM}$ (dotted line): $\Omega_{0}=0.3, \Omega_{\Lambda}=0.7$, $h=0.83, \Gamma=0.25$, and $\sigma_{8}=0.93$. Here the normalization factor $\sigma_{8}$ is determined from the X-ray cluster counts (Eke et al. 1996). It is clear that the redshift distribution of $\Omega_{0}=1$ models is drastically different from that of low $\Omega$ models. Because of the continuing growth of the linear density perturbation for the $\Omega_{0}=1$ models, their cluster numbers at high redshift (e.g., $z \geq 1$ ) are very tiny when the perturbations are normalized to the local cluster abundance. The presence of a few clusters at $z \geq 1$ would strongly falsify the $\Omega_{0}=1$ models. In fact, the existence of high-redshift optical or X-ray clusters has been used to constrain the range of $\Omega_{0}$ (e.g., Bahcall \& Fan 1998). On the other hand, the differences in the redshift distribution between the two lowdensity cosmological models are not as impressive as the differences between them and the $\Omega_{0}=1$ models, but they are still rather substantial at high redshifts. The number of

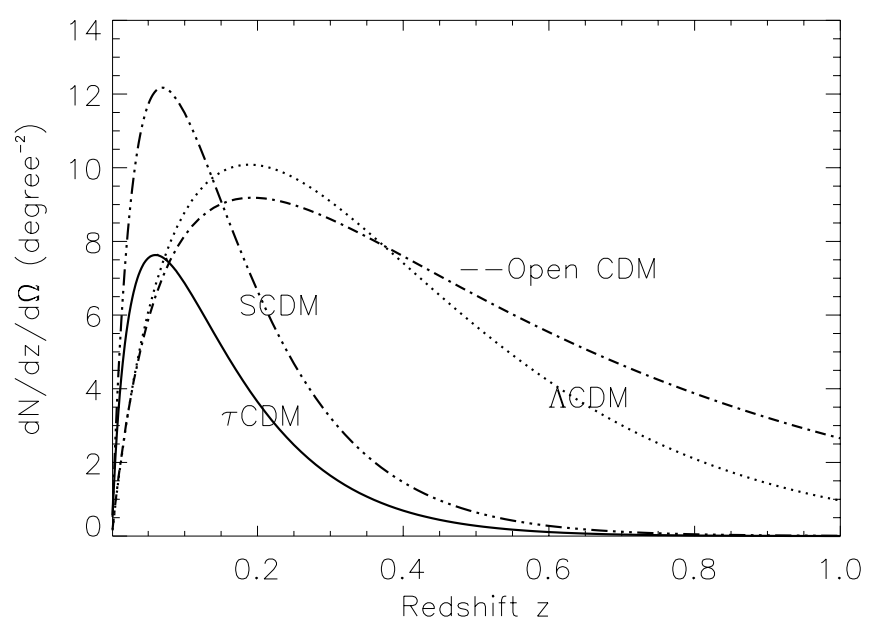

FIG. 1.-Redshift distributions of SZE clusters with $S_{v}^{\text {lim }} \approx 6.2 \mathrm{mJy}$. Solid line: $\tau \mathrm{CDM}$ model with $\Omega_{0}=1, h=0.5, \Gamma=0.25$, and $\sigma_{8}=0.52$. Dash-triple-dotted line: SCDM model with $\Omega_{0}=1, h=0.5, \Gamma=0.5$, and $\sigma_{8}=0.52$. Dash-dotted line: open CDM model with $\Omega_{0}=0.3, \Omega_{\Lambda}=0$, $h=0.83, \Gamma=0.25$, and $\sigma_{8}=0.87$. Dotted line: $\Lambda$ CDM model with $\Omega_{0}=$ $0.3, \Omega_{\Lambda}=0.7, h=0.83, \Gamma=0.25$, and $\sigma_{8}=0.93$. 
SZE clusters drops rapidly for the $\Lambda$ CMD model, while the redshift distribution for the low-density open model has a long tail at high redshifts. The different behaviors of the two models are mainly caused by the different angular diameter distances $R_{d}$ (see eq. [8]).

In the following discussion we study the ratio of the lowand high-redshift SZE clusters for the two types of lowdensity cosmologies. Specifically, the ratio $r=N(z \leq 0.5)$ $N(z \geq 1)$ is considered. The relative number of high- and low-redshift clusters with masses above a given threshold has been used in separately determining $\Omega_{0}$ and $\sigma_{8}$ by Fan, Bahcall, \& Cen (1997). Their study differs from ours in several respects, but both analyses take advantage of the dependence of the cluster evolution on cosmologies.

The model with $\Omega_{0}=0.3, \Omega_{\Lambda}=0.7, \Gamma=0.25$, and $\sigma_{8}=$ 0.93 is chosen to be the fiducial one. The particular $\sigma_{8}$ value is from the observed X-ray cluster counts. For other models, the cosmological density parameter is taken to be $0.25 \leq$ $\Omega_{0} \leq 0.35$. We vary $\Gamma$ in the range of $0.2 \leq \Gamma \leq 0.3$, consistent with large-scale structure studies (Peacock \& Dodds 1994; Dodelson \& Gaztanaga 2000).

We determine $\sigma_{8}$ of a specific model in such a way that it gives rise to about the same total number of flux-limited SZE clusters as that of the fiducial one at $S_{v}^{\text {lim }} \approx 6.2 \mathrm{mJy}$. We refer to this as SZE cluster-normalized $\sigma_{8}$. For the moment we pretend not to know the X-ray or optical cluster normalization (except for the $\sigma_{8}$ of the fiducial model), and only the SZE cluster counts are used in determining both $\sigma_{8}$ and $r$. In fact, the derived $\sigma_{8}$ from SZE cluster counts is consistent with that from X-ray or optical cluster counts for the parameter range we consider. However, below we see that if we require the same total number of SZE clusters for two models with significantly different values of $\Omega_{0}$ (e.g., $\Omega_{0}=0.2$ versus $\Omega_{0}=0.4$ ), at least one of the two $\sigma_{8}$ will have to be outside the current $\mathrm{X}$-ray cluster constraint. This inconsistency can be used in turn to falsify different models.

In Figure 2 we show $r$ versus $S_{v}^{\lim }$ for the fiducial cosmology (solid line), and for low-density open cosmologies. To avoid crowding, for open cosmologies only the highest and

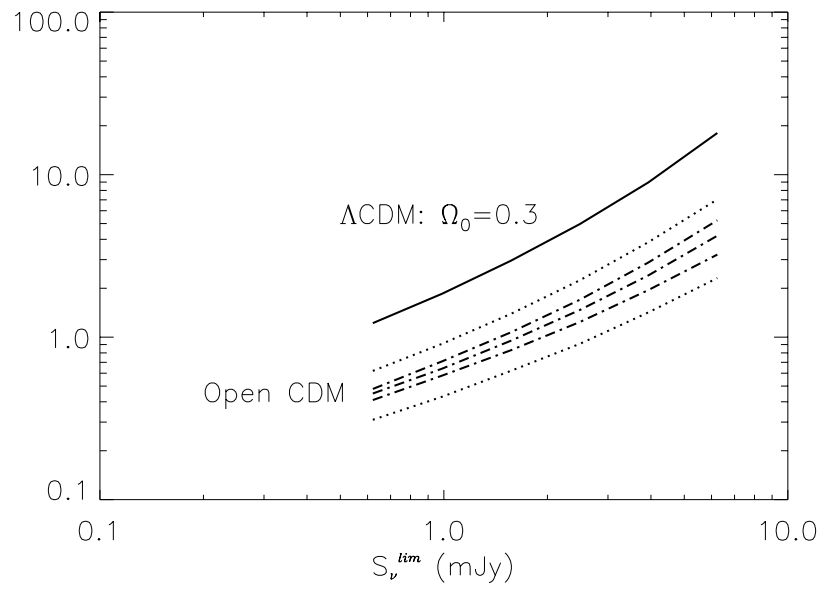

FIG. 2.-The ratio $r$ against the flux limit $S_{v}^{\text {lim }}$. Solid line: fiducial model with $\Omega_{0}=0.3, \Omega_{\Lambda}=0.7, \Gamma=\Omega_{0} h=0.25$, and $\sigma_{8}=0.93$. Top dotted line: open model with $\Omega_{0}=0.35, \Gamma=\Omega_{0} h=0.3$, and $\sigma_{8}=0.785$; bottom dotted line: open model with $\Omega_{0}=0.25, \Gamma=\Omega_{0} h=0.2$, and $\sigma_{8}=0.895$. The three dash-dotted lines are for $\Omega_{0}=0.3$ open models with $\Gamma=\Omega_{0} h=$ $0.3,0.25$, and 0.2 , and the corresponding $\sigma_{8}=0.79,0.835$, and 0.89 , from top to bottom. the lowest $r$ (dotted lines) for $0.25 \leq \Omega_{0} \leq 0.35$ and $0.2 \leq \Gamma \leq 0.3$ and the results for $\Omega_{0}=0.3$ with $\Gamma=0.2$, 0.25 , and 0.3 (dashed lines) are plotted. We can see that the $r$ range for the open universes is quite separate from that of the fiducial model. At $S_{v}^{\mathrm{lim}} \approx 6.2 \mathrm{mJy}, d N(z \leq 0.5) / d \Omega \approx$ $4.0 \mathrm{deg}^{-2}$ and $d N(z \geq 1) / d \Omega \approx 0.22 \mathrm{deg}^{-2}$ for the lowdensity flat fiducial model. For a SZE cluster survey that covers $50 \mathrm{deg}^{2}, N(z \leq 0.5) \approx 200$ and $N(z \geq 1) \approx 11$. Consider the Poisson noise; then $\sigma_{r} / r \approx(1 / 200+1 / 11)^{1 / 2} \approx$ 0.31 , where $\sigma_{r}$ is the standard deviation of $r$. Thus, the largest $r$ for the set of open universes is about $2 \sigma$ away from the fiducial $r$. The open model with $\Omega_{0}=0.3$ and $\Gamma=0.25$ is about $3 \sigma$ away. This demonstrates that if the $r$ value and the total number of SZE clusters from observations are indeed around the values of the fiducial model, low-density open models can be excluded at the 2-3 $\sigma$ level depending on how well the $\Omega_{0}$ and $\Gamma$ parameters have been determined. With larger surveys, the exclusion can be made with higher statistical significance. We emphasize that different models are SZE cluster normalized, and thus the above conclusion is independent of the "real" normalization (where "real" normalization means the conventional normalization from X-ray/optical cluster observations). On the other hand, since the total number of SZE clusters is sensitive to the normalization factor, the combined analysis of X-ray cluster counts and SZE cluster counts could falsify cosmological models if the normalizations determined separately from the X-ray clusters and from the SZE clusters disagree. This point is elaborated in the next subsection.

Attentive readers may have suspected that if one decreases $\Omega_{0}$ and $\Gamma$ for a flat model, its $r$ range can get closer to those of open models. In Figure 3, we show the result for $\Omega_{0}=0.25, \Omega_{\Lambda}=0.75, \Gamma=0.2$, and $\sigma_{8} \approx 1.01$ (model 1; solid line), along with some of the results for open models: $\Omega_{0}=0.35$ and $\Gamma=0.3(\operatorname{model} 2 ;$ dotted line $) ; \Omega_{0}=$ 0.25 and $\Gamma=0.3$ (model 3 ; upper dashed line); $\Omega=0.25$ and $\Gamma=0.25$ (model 4; middle dashed line); and $\Omega_{0}=0.25$ and $\Gamma=0.2$ (model $5 ;$ lower dashed line). All other open models with $0.25 \leq \Omega_{0} \leq 0.35$ and $0.2 \leq \Gamma \leq 0.3$ have $r$ values between the results of models 2 and 5 . The open models have been normalized so that they contain about the same

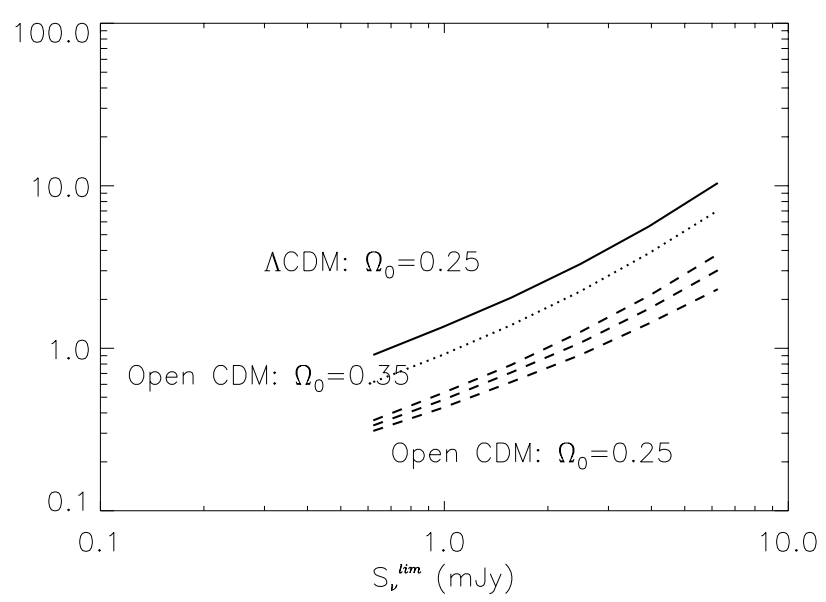

FIG. 3.-Plot of $r$ versus $S_{v}^{\mathrm{lim}}$. Solid line: $\Lambda \mathrm{CDM}$ model with $\Omega_{0}=0.25$, $\Omega_{\Lambda}=0.75, \Gamma=\Omega_{0} h=0.2$, and $\sigma_{8} \approx 1.01$ (model 1). Dotted line: open model with $\Omega_{0}=0.35, \Gamma=\Omega_{0} h=0.3$, and $\sigma_{8}=0.785$ (model 2). The three dashed lines are for the open models with $\Omega_{0}=0.25$, and from top to bottom $\Gamma=\Omega_{0} h=0.3,0.25$, and 0.2 , respectively (models 3,4 , and 5). The respective $\sigma_{8}$ for the open models with $\Omega_{0}=0.25$ are $0.795,0.84$, and 0.895 . 
total number of SZE clusters as model 1 at $S_{v}^{\mathrm{lim}} \approx 6.2 \mathrm{mJy}$. At this $S_{v}^{\text {lim }}$, model 1 has $r \approx 10.4$ and model 2 has $r \approx 7.0$. For model $1, d N(z \leq 0.5) / d \Omega \approx 3.6 \mathrm{deg}^{-2}$ and $d N(z \geq 1) /$ $d \Omega \approx 0.35 \mathrm{deg}^{-2}$, and the standard deviation of $r$ for a 50 $\operatorname{deg}^{2}$ survey is then $\sigma_{r} \approx 2.6$. Thus, model 2 differs from model 1 at about the $1 \sigma$ level. This difference itself may not be large enough to distinguish between the two models. We note, however, that the two parameters $\Omega_{0}$ and $\Gamma$ for the two models lie toward the opposite limit of our considered range: $\Omega_{0}=0.25$ and $\Gamma=0.2$ for model 1 , and $\Omega_{0}=0.35$ and $\Gamma=0.3$ for model 2. Hence, if $\Omega_{0}$ or $\Gamma$ can be constrained to better degrees by other observations, the lowdensity flat model and open models can be distinguished at a higher level of significance (for example, the Sloan Digital Sky Survey would give a better constraint on the $\Gamma$ parameter). This can be seen from the $r$ differences at $S_{v}^{\text {lim }} \approx$ $6.2 \mathrm{mJy}$, which are at 1.9 and $2.3 \sigma$ levels between model 1 and model 3, and between model 1 and model 4, respectively. Most drastically, model 1 and model 5 have the same $\Omega_{0}$ and $\Gamma$, and the difference in $r$ increases to the $3 \sigma$ level.

We show in Figure 4 the $\Gamma$ dependence of $r$ for $\Omega_{0}=0.3$ and $\Omega_{\Lambda}=0.7$. The three curves are for $\Gamma=0.2,0.25$, and 0.3 , respectively. All the models are normalized to contain about the same SZE clusters as our fiducial model at $S_{v}^{\text {lim }} \approx$ $6.2 \mathrm{mJy}$. It can be seen that $r$ is not very sensitive to $\Gamma$. In other words, $\Gamma$ cannot be strongly constrained by the SZE cluster counts alone. It is interesting to note that if the three models are all normalized to $\sigma_{8}=0.93$, the trend in $r$ is reversed from that shown in Figure 4, i.e., $r$ is largest for $\Gamma=0.2$, and smallest for $\Gamma=0.3$. However, the curves occupy the same region as that in Figure 4.

To see the $\Omega_{0}$ dependence of $r$, we plot $r$ in Figure 5 for low-density flat models with $\Omega_{0}=0.25,0.3$, and 0.35 . The $\Gamma$ parameter is taken to be 0.25 for all three models, and as before, they are normalized according to the total number of SZE clusters of the fiducial model at $S_{v}^{\lim } \approx 6.2 \mathrm{mJy}$. With such normalizations, $r$ is not sensitive to $\Omega_{0}$ either.

We conclude that $r$ is a very useful quantity in differentiating the low-density flat cosmological models from the low-density open cosmological models. Within the likely parameter regime of $\Omega_{0}$ and $\Gamma$, the quantity $r$ is not sensitive to either of them if different models are normalized consistently to the SZE cluster counts. This method is independent of the means by using the CMB measurement, and

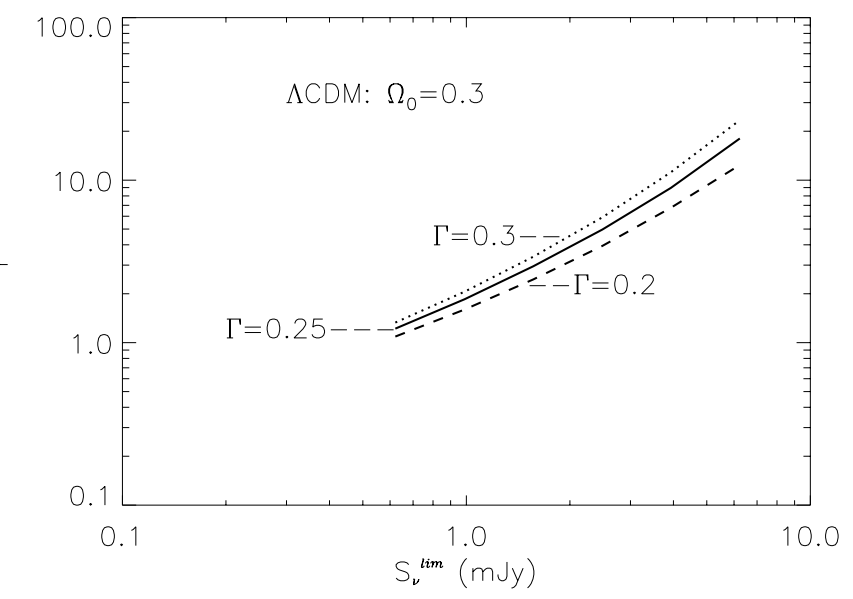

FIG. 4.-Plot of $r$ vs. $S_{v}^{\lim }$ for $\Omega_{0}=0.3$ and $\Omega_{\Lambda}=0.7$. Solid line: $\Gamma=$ $\Omega_{0} h=0.25$ and $\sigma_{8}=0.93$. Dotted line: $\Gamma=\Omega_{0} h=0.3$ and $\sigma_{8}=0.88$. Dashed line: $\Gamma=\Omega_{0} h=0.2$ and $\sigma_{8}=0.99$.

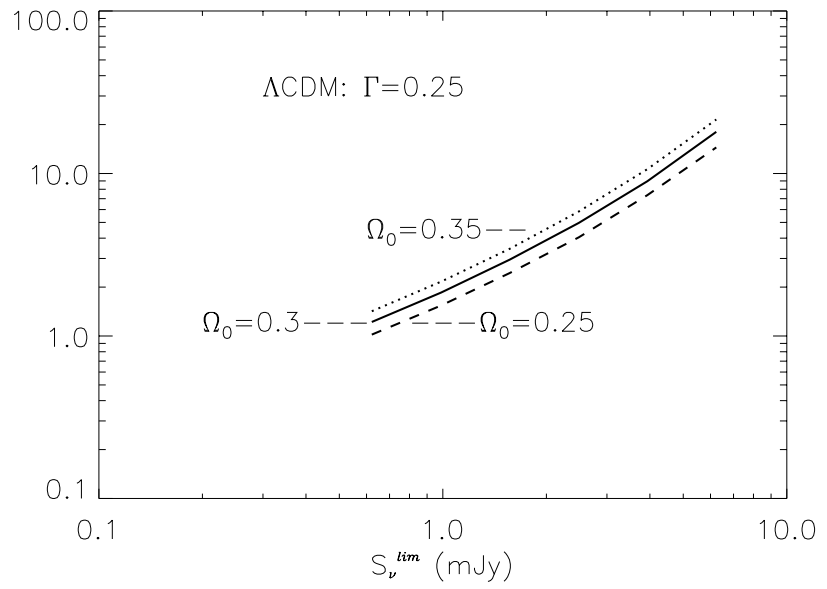

FIG. 5.-Plot of $r$ vs. $S_{v}^{\text {lim }}$ for $\Lambda$ CDM models with $\Gamma=\Omega_{0} h=0.25$. Solid line: $\Omega_{0}=0.3, \Omega_{\Lambda}=0.7$, and $\sigma_{8}=0.93$. Dotted line: $\Omega_{0}=0.35, \Omega_{\Lambda}=$ 0.65 , and $\sigma_{8}=0.91$. Dashed line: $\Omega_{0}=0.25, \Omega_{\Lambda}=0.75$, and $\sigma_{8}=0.95$.

thus provides an important test of our understanding of the universe and the formation of the large-scale structure.

Our conclusion above is not sensitive to the specific total number density of SZE clusters used to normalize different models. For instance, if we increase the total SZE cluster number density from 5.6 to $8.0 \mathrm{deg}^{-2}$ (the corresponding change of $\sigma_{8}$ of the fiducial model is from 0.93 to 1.0), the $\Lambda \mathrm{CDM}$ model with $\Omega_{0}=0.3$ and $\Gamma=0.25$ and the open model with the same $\Omega_{0}$ and $\Gamma$ can also be distinguished at about the $3 \sigma$ level for a $50 \mathrm{deg}^{2}$ survey. The operational steps to apply our method to observations are (1) to normalize different models to the observed total SZE cluster counts; (2) to calculate the $r$ value for different models; (3) to find the $r$ value from observations; and (4) to compare the results of step 3 with those of step 2.

On the other hand, the relatively large separation of the $r$ value between the $\Lambda \mathrm{CDM}$ and the open CDM models shown above is restricted to our considered parameter intervals of $\Omega_{0}$ and $\Gamma$. If the $\Omega_{0}$ range is increased to $0.2 \leq$ $\Omega_{0} \leq 0.4$, there will be some overlaps in $r$ for the two types of models. For example, the $r$ value of the $\Lambda$ CDM model with $\Omega_{0}=0.2$ falls into about the same range as that of the open CDM model with $\Omega_{0}=0.4$. Thus, with only the SZE total counts and the $r$ value, the two models cannot be differentiated clearly. However, in order to have the same total number of SZE clusters, at least one of the two $\sigma_{8}$ must be out of the range allowed by the observed X-ray cluster counts. Therefore, an exclusion is possible based on both the SZE cluster observations and the X-ray observations. Stated somewhat differently, in order to make a relatively clean distinction between the $\Lambda \mathrm{CDM}$ and the open CDM models by using $r$ alone, the parameters $\Omega_{0}$ and $\Gamma$ must be predetermined by other observations to a relatively fine degree. In conjunction with X-ray or optical cluster observations, the SZE cluster counts can be used to distinguish the two types of models for wider parameter regimes.

\subsection{Constraints on $\Omega_{0}$ and $\sigma_{8}$ for a Flat Universe}

Results from the recent Boomerang CMB observation show the first Doppler peak at $l \approx 200$ beautifully (de Bernardis et al. 2000), and have made the flat universe widely accepted (Hu 2000a, 2000b; Tegmark \& Zaldarriaga 2000). 
If we do indeed live in a flat universe with a nonzero cosmological constant, then, as we describe below, the combined analyses of X-ray cluster counts and SZE cluster counts can give rise to constraints on $\Omega_{0}$ and $\sigma_{8}$ even without the cluster redshift information.

The key here is that the dependence of $\sigma_{8}$ on $\Omega_{0}$ inferred from X-ray cluster counts is different from that inferred from SZE cluster counts. The X-ray cluster counts yielded the relation $\sigma_{8}=(0.52 \pm 0.04) \Omega_{0}^{-0.52+0.13 \Omega_{0}}$ for $\Omega_{0}+$ $\Omega_{\Lambda}=1$ (Eke et al. 1996). Here we study the expected $\sigma_{8}-\Omega_{0}$ correlation from SZE cluster counts.

The formulation presented in $\S 2$ is used to study the SZE $\sigma_{8}-\Omega_{0}$ relation. Specifically, the following assumptions are employed: (1) the intracluster gas is in hydrostatic equilibrium in the gravitational potential well of the total cluster mass; (2) the gas is isothermal and the gas mass fraction is a constant among different clusters; (3) the collapse is approximately spherical; and (4) the Press-Schechter formula is approximately correct in predicting the number of clusters. We would like to point out that the same approximations have been used in deriving the $\sigma_{8}-\Omega_{0}$ correlation from X-ray cluster observations (Eke et al. 1996)

The model with $\Omega_{0}=0.3, \Omega_{\Lambda}=0.7, \Gamma=0.25$, and $\sigma_{8} \approx$ 0.93 is taken to be the fiducial one. We first compute the total surface number density of SZE clusters at $S_{v}{ }_{v}$ im $=6.2$ $\mathrm{mJy}$ for the fiducial model, and then find $\sigma_{8}$ values for other $\Lambda \mathrm{CDM}$ models with different $\Omega_{0}$ so that they have the same total surface number density of SZE clusters at $S_{v}{ }^{\lim }=6.2$ $\mathrm{mJy}$ as the fiducial model.

The results are shown in Figure 6. We find that the relation can be nearly perfectly described by $\sigma_{8}=A_{S} \Omega_{0}^{-0.13}$ (Fig. 6, solid line), where $A_{S}$ is a numerical factor that is equal to 0.794 in our analysis. The dependence of the SZE cluster-normalized $\sigma_{8}$ on $\Omega_{0}$ is much weaker than that of the X-ray cluster-normalized $\sigma_{8}$, which can be understood from equations (7) and (8). The X-ray cluster-normalized $\sigma_{8}$ is calculated from temperature-limited cluster counts (Eke et al. 1996). The cluster mass limit, $M_{\text {lim }}$, derived from a given cluster gas temperature threshold is proportional to $\left[\Omega_{0} / \Omega(z)\right]^{-1 / 2} \Delta_{c}^{-1 / 2}$ (eq. [7]). By contrast, given an SZ flux

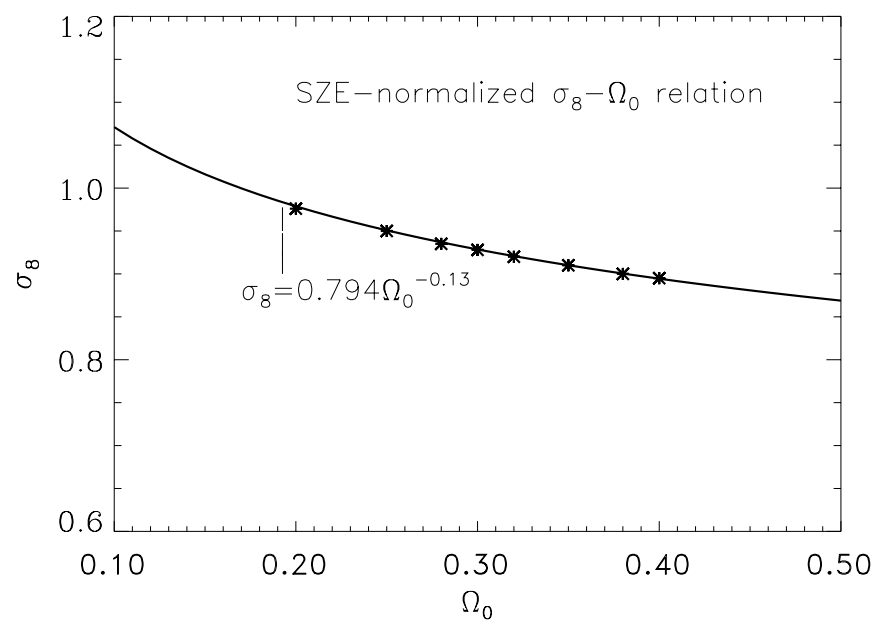

FIG. 6.-SZE cluster-normalized $\sigma_{8}$ as a function of $\Omega_{0}$ for flat universes with a nonzero cosmological constant. The asterisks show the numerical results calculated by requiring that models with different $\Omega_{0}$ contain the same number of SZE clusters at $S_{v}^{\mathrm{lim}} \approx 6.2 \mathrm{mJy}$ as that of the fiducial model. The fitting relation $\sigma_{8}=0.794 \Omega_{0}^{-0.13}$ is shown by the solid line. limit $S_{v}^{\text {lim }}$, we have $M_{\text {lim }} \propto\left[\Omega_{0} / \Omega(z)\right]^{-1 / 5} \Delta_{c}^{-1 / 5}$ (eq. [8]). Since the cluster number counts from the Press-Schechter formalism are sensitive to the mass limit, the $\sigma_{8}$ inferred by comparing results from X-ray observations with the predictions of the Press-Schechter calculations has a dependence on $\Omega_{0}$ that differs from that found from SZE cluster "observations."

The total number density of SZE clusters for the fiducial model at $S_{v}^{\mathrm{lim}} \approx 6.2 \mathrm{mJy}$ is $d N(z \geq 0) / d \Omega \approx 5.6 \mathrm{deg}^{-2}$. With a $50 \mathrm{deg}^{2}$ survey, the total number of SZE clusters is expected to be about 200, and the standard deviation given by the Poisson statistics is then about 16.7. Thus the $3 \sigma$ number density is $5.6 \pm 1.0 \mathrm{deg}^{-2}$. The corresponding $\sigma_{8}$ range is calculated, which can be well approximated by $\sigma_{8}=(0.794 \pm 0.025) \Omega_{0}^{-0.13}$. In Figure 7 , we plot the $\sigma_{8}-\Omega_{0}$ relations expected both from the X-ray observations (dashed lines) and from our analysis of the SZE clusters (solid lines). Based on the current X-ray cluster results and our proposed $50 \mathrm{deg}^{2}$ SZE cluster survey, the fiducial $\Omega_{0}$ can be determined to $\Omega_{0}=0.3 \pm 0.08$ or $\Omega_{0}=(1 \pm 27 \%)$ $\times 0.3$. From Figure 7, it can be seen that the $X$-ray cluster normalization more tightly constrains the value of $\Omega_{0}$, since it is much more sensitive to $\Omega_{0}$. With future X-ray cluster surveys such as $X M M$, the normalization can be determined to a much better degree. At a $2 \%$ precision on $\sigma_{8}$ from X-ray surveys, $\Omega_{0}$ can be constrained to $0.3 \pm 0.04$ ( $\sim 13 \%$ precision) with the allowance of a $3 \sigma$ deviation of SZE cluster counts. Comparing with the results of Haiman et al. (2000; see their Fig. 7) for the $w=-1$ case, we find that their constraint on $\Omega_{0}$ is more stringent. However, in their analyses they normalize all models to give rise to the local cluster number density with $M \geq 10^{14} h^{-1} M_{\odot}$; i.e., for each model, the normalization is fixed. They then compare both the total number of SZE clusters and the redshift distribution of a model with those of their fiducial model to constrain the parameter regimes. Note that for the $w=-1$ case, the total number of SZE clusters plays the dominant role in constraining $\Omega_{0}$. If we focus on the middle dashed line in our Figure 7, the $3 \sigma$ determination of $\Omega_{0}$ from the SZE total number counts is similar to that of Haiman et al. (2000). Be aware, however, of the different

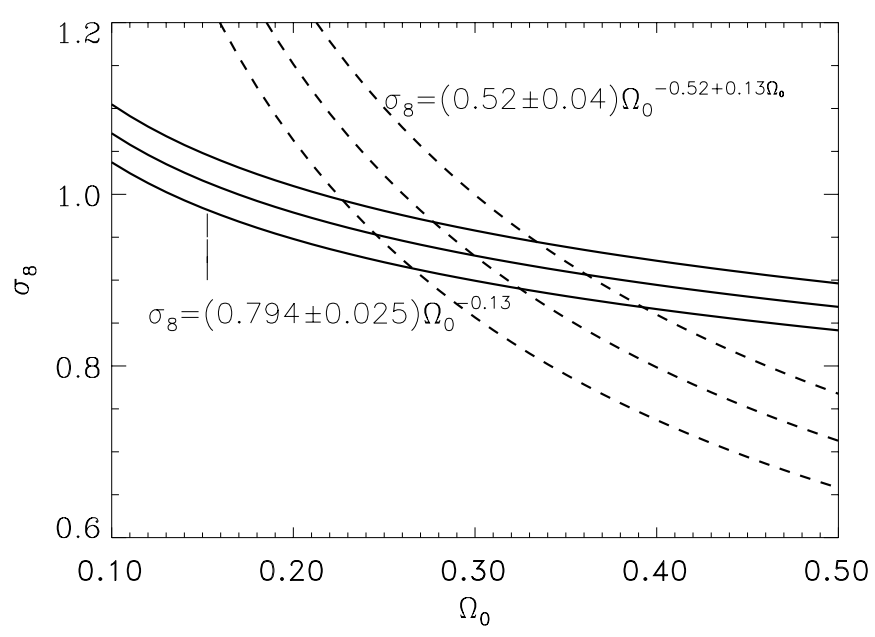

FIG. 7.-Plot of $\sigma_{8}$ vs. $\Omega_{0}$ for flat universes. The solid lines are from the SZE cluster counts, and from top to bottom, $\sigma_{8}=0.819 \Omega_{0}^{-0.13}$, $0.794 \Omega^{-0.13}$, and $0.769 \Omega^{-0.13}{ }_{0}$, respectively. The dashed lines are from $\mathrm{X}$-ray temperature-limited cluster counts, and $\sigma_{8}=0.56 \Omega_{0}^{-0.52+0.13 \Omega_{0}}$, $0.52 \Omega_{0}^{-0.52+0.13 \Omega_{0}}$, and $0.48 \Omega_{0}^{-0.52+0.13 \Omega_{0}}$ (top to bottom). 
cosmological parameters, normalizations, and survey parameters used in their analyses and in our studies.

The normalization factor $\sigma_{8}$ can also be constrained at the same time. In contrast to the determination of $\Omega_{0}$, the $\sigma_{8}$ value is constrained more tightly by the SZE cluster counts. At $\Omega_{0}=0.3$, the $3 \sigma$ determination of $\sigma_{8}$ is $0.93 \pm 0.03$ or $(1 \pm 3.2 \%) \times 0.93$, compared to $(1 \pm 7.5 \%) \times 0.93$ from the current X-ray studies. If the allowed range of $\Omega_{0}$ is $0.3 \pm 0.08, \sigma_{8}$ can be constrained to $0.93 \pm 0.063$, or $\sigma_{8} \sim(1+6.7 \%) \times 0.93$.

We emphasize that although we chose $\Gamma=0.25$ in the above analysis, calculations have been done for other $\Gamma$ values. It has been found that the functional relation $\sigma_{8} \propto$ $\Omega_{0}^{-0.13}$ is very insensitive to the $\Gamma$ value. The power index of the $\sigma_{8}=\Omega_{0}$ relation has a weak dependence on $S_{v}^{\text {lim }}$ : the smaller the $S_{v}^{\lim }$, the flatter the power-law relation. For example, the index equals -0.14 and -0.11 for $S_{v}^{\text {lim }}=$ $10 \mathrm{mJy}$ and $3 \mathrm{mJy}$, respectively. The constraints on $\Omega_{0}$ and $\sigma_{8}$, however, change little by this index variation.

\section{SUMMARY}

In our analyses, we used $f_{\text {ICM }}=0.1, X=0.76$, and $\left[d \ln \rho_{\mathrm{gas}}(r) / d \ln r\right]_{r_{\mathrm{vir}}}=2$. To change these parameters, however, is equivalent to changing the overall flux limit $S_{v}^{\mathrm{lim}}$. From our figures, it is easy to see that all our conclusions remain qualitatively unchanged for different choices of these parameters. We have assumed hydrodynamic equilibrium and isothermality for the intracluster gas. The Press-Schechter formalism has been adopted to calculate the cluster counts.

We studied the $r$ quantity for two types of cosmologies: the low-density flat models and the low-density open models. Within the studied parameter regimes $0.25 \leq \Omega_{0} \leq$ 0.35 and $0.2 \leq \Gamma \leq 0.3$, the $r$ values for the two sets of cosmologies are well separated. The flat model with $\Omega_{0}=0.3$ and $\Gamma=0.25$ and the open model with the same $\Omega_{0}$ and $\Gamma$ can be differentiated at the $3 \sigma$ level for a $50 \mathrm{deg}^{2}$ survey. Since we normalize different models in such a way that they give rise to the same total number of SZE clusters, our analyses naturally take into account the total number of SZE clusters. For wider parameter ranges, the information from other observations, such as X-ray or optical cluster surveys, must be used to differentiate the two types of models.

There are other ways to determine the geometry of the universe. Measurements of fluctuations of the CMB radi- ation provide a clean test of this aspect (e.g., $\mathrm{Hu} 2000 \mathrm{a}$, $2000 \mathrm{~b}$ ). The horizon size of the universe at decoupling separates large-scale and small-scale CMB fluctuations. Large-scale fluctuations were outside the horizon when photons escaped, while small-scale perturbations were within the horizon at decoupling and therefore sustained acoustic oscillations. The position of the primary Doppler peak of the CMB fluctuation power spectrum is determined by the angular size of the horizon at decoupling. For a flat universe (low-density with a nonzero cosmological constant or high density), the peak is located at $l \approx 200$, and the peak is shifted to smaller angular scale or higher $l$ for a lowdensity open universe, where $l$ represents the twodimensional angular wavenumber. Observations have seen rising and declining CMB fluctuations around $l \sim 200$ (e.g., Miller et al. 1999; de Bernardis et al. 2000), which convincingly constrains $\Omega_{\text {tot }}$ to be close to 1 , where $\Omega_{\text {tot }}$ is the total density parameter. Combined with other information, e.g., supernova measurements (Perlmutter et al. 1999; Schmidt et al. 1998), this leads to the conclusion that we are living in a low-density flat universe. If the results from SZE cluster surveys are in agreement with the CMB results, we will be in a more solid position to say that the universe is flat and that the simple structure formation theory we have adopted here is reasonably correct. On the other hand, any inconsistency between the SZE cluster results and the results from CMB measurements would pose challenges to our understanding of the universe. For example, alternate structure formation theories, such as non-Gaussian initial fluctuation models, may need to be considered.

Should the universe be flat, SZE cluster surveys can also provide constraints on cosmological parameters. We studied $\sigma_{8}\left(\Omega_{0}\right)$ inferred from the total SZE cluster counts. A functional relation $\sigma_{8} \propto \Omega^{-0.13}$ is found. Combined with the current X-ray cluster-normalized $\sigma_{8}$, the parameters can be determined (taking $\Omega_{0}=0.3$ and $\sigma_{8}=0.93$ as the central values) to $\Omega_{0}=(1 \pm 27 \%) \times 0.3$ and $\sigma_{8}=$ $(1 \pm 6.7 \%) \times 0.93$ for the SZE cluster counts confined to the $3 \sigma$ level in a $50 \mathrm{deg}^{2}$ survey. Note that these constraints are from the total number of clusters only, and no redshift information is needed.

We thank the referee for constructive comments and $\mathrm{H}$. Liang for valuable discussions. This research was supported in part by grants NSC89-2112-M002-037 and NSC89-2816M-001-0006-6 from the National Science Council, R.O.C.
Bahcall, N., \& Fan, X. 1998, ApJ, 504, 1

Birkinshaw, B. 1999, Phys. Rep., 310, 97

Carlstrom, J., et al. 2000, Phys. Scr., 85, 148

de Bernardis, P., et al. 2000, Nature, 404, 955

Dodelson, S., \& Gaztanaga, E. 2000, MNRAS, 312, 774

Efstathiou, G., Bond, J. R., \& White, S. D. M. 1992, MNRAS, 258, 1 P

Eke, V. R., Cole, S., \& Frenk, C. S. 1996, MNRAS, 282, 263

Fan, X. H., Bahcall, N. A., \& Cen, R. 1997, ApJ, 490, L123

Grego, L., et al. 2000, ApJ, 539, 39

Haiman, Z., Mohr, J. J., \& Holder, G. P. 2000, ApJ, submitted (preprint astro-ph/0002336)

Holder, G. P., Mohr, J. J., Carlstrom, J. E., Evrard, A. E., \& Leitch, E. M. 2000, ApJ, 544, 629

$\mathrm{Hu}, \mathrm{W} .2000 \mathrm{a}$, preprint (astro-ph/0002520)

Hu, W. 2000b, Nature, 404, 939

\section{REFERENCES}

Miller, A. D., et al. 1999, ApJ, 524, L1

Mohr, J., Carlstrom, J., Holder, G., Hozapfel, W., Joy, M., Leitch, E., \& Reese, E. 2000, in From Extrasolar Planets to Cosmology, ed. J. Bergeron \& A. Renzini (Berlin: Springer), 150

Navarro, J. F., Frenk, C. S., \& White, S. D. M. 1997, ApJ, 490, 493

Peacock, J., \& Dodds, S. 1994, MNRAS, 267, 1020

Perlmutter, S., et al. 1999, ApJ, 517, 565

Press, W., \& Schechter, P. 1974, ApJ, 187, 425

Reese, E. D., et al. 2000, ApJ, 533, 38

Schmidt, B. P., et al. 1998, ApJ, 507, 46

Sunyaev, R. A., \& Zeldovich, Ya. B. 1970, Comments Astrophys. Space Phys., 2, 66

. 1980, ARA\&A, 18, 537

Tegmark, M., \& Zaldarriaga, M. 2000, Phys. Rev. Lett., 85, 2240

White, M., Gelmini, G., \& Silk, J. 1995, Phys. Rev. D, 51, 2669 